\title{
THE SUBMILLIMETER TELESCOPE - A STATUS REPORT
}

\author{
R. N. MARTIN and J. W. M. BAARS \\ Submillimeter Telescope Observatory \\ University of Arizona, Tucson, AZ
}

\section{SUMMARY}

The Max-Planck-Institut für Radioastronomie, Bonn and the Steward Observatory of the University of Arizona, Tucson are collaborating on the construction and operation of a dedicated submillimeter facility. The Submillimeter Telescope (SMT) is a $10 \mathrm{~m}$ diameter reflector surrounded by a corotating enclosure. The instrument is an alt-azimuth mounted $\mathrm{f} / 13.8 \mathrm{Cassegrain}$ homology telescope with two Nasmyth and bent Cassegrain foci. The SMT will have diffraction limited performance at a wavelength of $300 \mu \mathrm{m}$ and an operating overall figure accuracy of $15 \mu \mathrm{m}$ rms. The primary and secondary reflector surfaces are constructed out of aluminum-core, carbon-fiber-reinforced-plastic (CFRP) face sheet sandwich panels. The primary reflector backup structure and secondary support are fabricated from CFRP structural members. This modern technology provides both the means for reaching the required precision of the SMT for both night and day operation (basically because of the low coefficient of thermal expansion and high strength-to-weight ratio of CFRP) and a potential route for the realization of lightweight telescopes of even greater accuracy in the future. The SMT will be the highest accuracy radio telescope ever built.

The SMT is located at an altitude of $3180 \mathrm{~m}$ on Emerald Peak (Mt. Graham) $120 \mathrm{~km}$ northeast of Tucson in southern Arizona. Measurements indicate that atmospheric conditions allow submillimeter observations during about $40 \%$ of the time in winter months. The telescope is placed in a co-rotating enclosure of novel design. The enclosure fits tightly around the telescope, with the focus flanges extending from the elevation bearings into the receiver rooms of the enclosure. A flat tertiary mirror is used to direct the beam through either of the two elevation bearings. Thus, we are able to mount receivers directly on the telescope while maintaining laboratory type conditions in the access area surrounding the receivers. On the "facility instrument" side, several receivers can be operated simultaneously.

The initial set of receivers is being developed in the laboratories of the MPIfR and the Steward Observatory. Spectroscopic receivers, based on SIS technology, are being constructed for the 350,460 and $670 \mathrm{GHz}$ atmospheric windows. Further systems, especially for higher frequencies, are being developed. For continuum observations, ${ }^{3} \mathrm{He}$ cooled bolometers will cover the major atmospheric windows. 
A more extensive description of this project is given by Martin and Baars (1990) and Baars and Martin (1990).

\section{STATUS}

Construction of the SMT enclosure began in 1991 on Mt. Graham. The enclosure is expected to be completed in spring 1993. The telescope reflector structure and mount have been fabricated by Krupp Industrietechnik in Germany. The reflector panels have been fabricated by MAN Technologie in Germany. The mount for the SMT was placed in the enclosure in October 1992. The erection of the reflector structure will commence in early 1993 and be completed by summer 1993. We then expect to begin the adjustment of the reflector surface and to begin the initial testing of the telescope system.

\section{REFERENCES}

Martin, R. N. and Baars, J. W. M. 1990, SPIE, 1235, 503

Baars, J. W. M. and Martin, R. N. 1990, Proc $29^{\text {th }}$ Liège Int Ast Coll, 293 